NOTA CIENTÍFICA

\title{
Evaluación de colores para la oviposición de Ceratitis capitata (Diptera, Tephritidae) en Argentina
}

\section{Evaluation of colours to oviposition of Ceratitis capitata (Diptera, Tephri- tidae) in Argentina}

\author{
Lorena Suárez ${ }^{1}$, Andrea Molina ${ }^{1}$, Fernando Murúa1, Juan Carlos Acosta ${ }^{2}$, \\ Blanca Moyano ${ }^{1}$ y Jorge Escobar ${ }^{1}$
}

${ }^{1}$ Procem San Juan - Dirección de Sanidad Vegetal, Av. Benavidez 8000 CP 5400 San Juan, Argentina.

Email Lorena Suarez:

lorenasuarez7@yahoo.com.ar

${ }^{2}$ Departamento de Biología e Instituto y Museo de Ciencias NaturalesFCEFyN, Universidad Nacional de San Juan, Argentina.

Presentado: 22/06/2006 Aceptado: $\quad 12 / 07 / 2007$

\section{Introducción}

Ceratitis capitata Wiedemann, Díptero de la Familia Tephritidae, es una de las plagas más importantes de la fruticultura mundial, atacando más de 250 especies vegetales (Quesada Allué, 1994).

En Argentina, constituye la especie de mosca de los frutos de mayor distribución e importancia (Blanchard, 1961) y está sometida a acciones de control bajo distintos programas regionales que utilizan la Técnica del Insecto Estéril (TIE) en el marco del Manejo Integrado de Plagas.

Para perfeccionar las técnicas de trampeo en los sistemas de detección de Mosca de los Frutos, distintos autores evaluaron materiales en diversas condiciones como sustrato de oviposición probando, en general, el efecto de la forma y color sobre la capturabilidad de C. capitata (Féron et al., 1958; Féron, 1962; Sanders 1962, 1968a y b; Tanaka, 1965; Nakagawa et al., 1978; Cytrynowicz et al., 1982; Marchini \& Wood, 1983; Prokopy et al., 1984; Freeman \& Carey 1990).

Numerosos estudios sostienen que los estímulos físicos poseen un papel muy importante en la localización y selección de los frutos hospederos en especial a corta distancia. Entre estos estímulos se mencionan la forma, tamaño, color, grado de contraste del fruto contra el fondo (Féron, 1962; Sanders, 1968b; Nakagawa et al., 1978; Cytrynowicz et al., 1982; Marchini \& Wood, 1983; Prokopy et al., 1984) y el matiz (Prokopy \& Economopoulos, 1975; Boller \& Prokopy, 1976; Owens \& Pro- kopy, 1984). En la proximidad de la fruta, a menos de un metro, dentro de la canopia del árbol, la atracción visual representa la fuerza predominante que conduce a la mosca hacia el fruto (Drummond et al., 1984). En consecuencia, la forma, el color y el tamaño de las hojas y frutos son de gran importancia para la detección y selección de los sitios disponibles para oviponer (Prokopy, 1977b).

Objetos inanimados pueden mimetizarse con los frutos cuando se suspenden sobre plantas hospederas, atrayendo así a las moscas de la fruta, resultando adecuados para propósitos de trampeo (Katsoyannos, 1989). El uso de trampas constituye una de las formas más utilizadas por los programas de control para evaluar el resultado de sus acciones. El desarrollo de una trampa efectiva de huevos, a base de un sustrato oviposición, puede ser una poderosa herramienta de monitoreo para hembras grávidas. Esto permitiría medir el grado de esterilidad inducida en la población bajo presión de TIE, ayudando así a evaluar el éxito de su aplicación.

El objetivo de este trabajo fue evaluar la capacidad atrayente de esferas de agar de distintos colores para la oviposición de cepas locales de C. capitata en un sustrato de agar, en condiciones de laboratorio.

\section{Materiales y métodos}

Los ensayos se realizaron en los laboratorios de la Bioplanta del Programa de Control y Erradicación de Mosca de los Frutos de San Juan (ProCEM). 
Tabla 1. Medias, rangos y errores estándar del número de huevos para cada tratamiento, en la evaluación de colores para la oviposición de Ceratitis capitata

\begin{tabular}{cccc}
\hline Colores & Media & Rango & Error Estándar \\
\hline Amarillo & 16,49 & 16,49 & 7 \\
Rojo & 57,66 & 74 & 8,33 \\
Verde & 38,63 & 57 & 5,51 \\
Negro & 49,19 & 52 & 4,86 \\
Sin Color & 10,83 & 18,67 & 1,70 \\
\hline
\end{tabular}

Las pupas de $C$. capitata colectadas a campo a partir de fruta infestada, se colocaron dentro de cajas de acrílico, en cámara climatizada a $23^{\circ} \mathrm{C}, 70 \% \mathrm{HR}$ y $12 \mathrm{hs}$ (L:O) hasta la emergencia de los adultos.

Los mismos, fueron separados por sexo y edad; de 9, 10, 11 y 12 días; y se acondicionaron en recipientes de vidrio de $900 \mathrm{~cm}^{3}$, hasta alcanzar la madurez sexual. Se los alimentó ad libitum, con la dieta estándar para la cría masiva de $C$. capitata: una mezcla de tres partes de azúcar refinada y una de proteína $\mathrm{ICN}^{\ominus}$.

En cajas de acrílico transparente $(0,40 \mathrm{~m}$ x $0,40 \mathrm{~m}$ x 0,40 $\mathrm{m})$, se colocaron individuos de ambos sexos a fin de inducir la cópula. A medida que ocurrían los apareamientos, cada pareja fue extraída en un tubo de ensayo, donde se mantuvo hasta finalizar la cópula.

Se prepararon 10 cajas (réplicas) iguales a las mencionadas, colocando 9 hembras en cada una. Se evaluaron 5 tratamientos, representados por los colores amarillo, rojo, verde, negro y sin color (o control). Se realizaron 3 repeticiones $(n=150)$ de cada tratamiento. Las esferas fueron colgadas dentro de las cajas a diferentes alturas, en un arreglo totalmente al azar.

Las esferas, de $4 \mathrm{~cm}$ de diámetro, se fabricaron utilizando: agua fría, agar-agar industrial, azúcar y colorante en pasta para repostería Circe ${ }^{\otimes}$, con excepción del tratamiento control, fabricado sólo con agar. Se utilizó benzoato de sodio para prevenir la

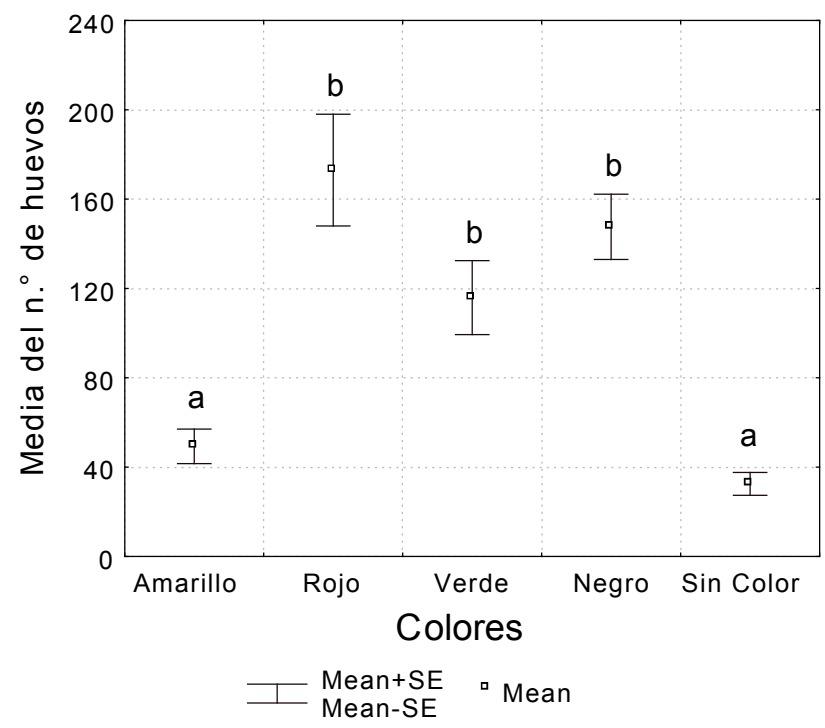

Figura 1. Media y error estándar del número de huevos depositados por las hembras en cada color, en la evaluación de colores para la oviposición de Ceratitis capitata. Letras diferentes indican diferencias estadísticas significativas según el Test LSD. formación de hongos. La deshidratación de las esferas se evitó con papel filme adherente Rolopac ${ }^{\oplus}$, agregándose un cordel de algodón como elemento de suspensión.

Las cajas se mantuvieron durante 8 días, en cámaras climatizadas a $23^{\circ} \mathrm{C}, 60-70 \% \mathrm{HR}$ y $12 \mathrm{~h}(\mathrm{~L}: \mathrm{O})$, con agua y comida ad libitum.

Las esferas de agar, se disectaron bajo lupa binocular, registrándose el número de oviposturas y de huevos depositados en cada una. Para ello, cada ovipostura, fue colocada en una caja de Petri sobre papel absorbente negro con agua. Mientras se producía la disolución del agar, los huevos liberados, fueron contados.

Los datos muestrales se trataron con estadísticos descriptivos, asegurándose su independencia y homocedaticidad mediante las pruebas de Kolmogorov-Smirnov y Levenne, respectivamente.

Se realizaron regresiones (cuadrados mínimos) y correlaciones (Pearson) para los parámetros: número de huevos y de oviposturas, en cada uno de los tratamientos.

\section{Resultados}

En el total de las muestras analizadas $(\mathrm{n}=150)$ se contaron 2082 oviposturas y 5185 huevos, con un promedio de entre 2 y 3 huevos por ovipostura.

Existe alta correlación $(r=0,88 ; \mathrm{p}=0,000001 ; \mathrm{n}=150)$ entre las variables número de huevos y oviposturas, considerando todos los tratamientos en conjunto. Por ello, se eligió al número de huevos para evaluar la capacidad atrayente de los distintos colores, en análisis posteriores.

Las hembras colocaron un promedio máximo de 57,66 huevos en las esferas rojas y un mínimo de 10,83 huevos en las sin color (Tabla 1). Se encontraron diferencias estadísticamente significativas entre tratamientos (ANOVA, $\mathrm{F}_{(4,45)}=15,54 ; \mathrm{p}=0,000001$ ).

Según el Test LSD las hembras depositaron una mayor cantidad de huevos en las esferas rojas, verdes y negras sobre las amarillas y sin color (Fig. 1).

Se encontraron diferencias estadísticamente significativas entre las cajas para todos los colores (ANOVA, $\mathrm{F}_{(45,74)}=2,00$; $\mathrm{p}=0,004 ; \mathrm{n}=10)$.

\section{Discusión}

Las moscas frugívoras detectan los frutos hospederos, a través de sus características físicas, tales como forma, tamaño, intensidad de refractancia y grado de contraste con el fondo. Por otro lado la literatura sobre ensayos con trampas para adultos en laboratorio, con fines de control o monitoreo, arroja resultados contradictorios.

Sanders (1968b) reportó que las hembras, criadas en laboratorio, expuestas a diferentes esferas coloreadas ubicadas sobre un fondo monocromático, oviponen en aquellas que muestran mayor contraste contra el fondo. Sin embargo, cuando los mismos modelos de frutos son colocados sobre un fondo dicromático (negro y banco o negro y gris), las hembras prefieren la parte oscura sin tener en cuenta el grado de contraste que éste posea. Este autor concluyó que el color del fondo es más importante que el grado de contraste.

Souza et al. (1984), observaron que hembras de C. capitata eligen oviponer en esferas de agar rojas sobre las verdes y amaril- 
las. Por otra parte, Marchini \& Wood (1983) observaron que el amarillo es más atractivo para hembras de C. capitata, seguido por el verde y rojo. Esta preferencia es similar a la observada para otras especies como C. rosa y Bactrocera oleae por Quilici \& Rivry (1996) y Katsoyannos, et al. (1985), respectivamente.

En el presente estudio, la cantidad de huevos depositados en las esferas de agar rojas, negras y verdes fue significativamente mayor que en las amarillas y sin color; esto podría sugerir que las hembras se ven más atraídas hacia los colores oscuros. Estos resultados coinciden con las observaciones hechas por Sanders (1968 b) y Souza et al. (1984) y resultan contradictorios con lo reportado por Marchini \& Wood (1983) y Katsoyannos et al. (1985).

En una revisión reciente, Katsoyannos (1989) remarca la importancia de la presencia de hormonas disuasoras de la oviposición, como un factor que afecta la respuesta de la hembra frente a los sitios de oviposición, en varias especies de moscas, incluyendo C. capitata. Sin embargo, en nuestro experimento, los colores preferidos lo continuaron siendo, aún cuando el número de huevos depositados fue muy alto y también lo fue, la concentración de la hormona mencionada.

Podemos concluir que, en condiciones de laboratorio:

-Las esferas de agar son útiles como sustratos de oviposición para hembras de Ceratitis capitata.

-Las hembras de Ceratitis capitata son capaces de diferenciar colores durante la selección de los sitios de oviposición.

-Las hembras fueron atraídas hacia los colores rojo y negro, lo cual se debería a un efecto de alto contraste de estos colores contra el fondo.

\section{Literatura citada}

Blanchard E. E. 1961. Especies argentinas del género Anastrepha Schiner (sens. Lat.) (Diptera: Trypetidae). Revista de Investigaciones Agrícolas XV (2): 281-342.

Boller E. F. \& R. J. Prokopy. 1976. Bionomics and management of Rhagoletis. Annual Review of Entomology 21: 223-246.

Cytrynowicz M., J. S. Morgante \& H. M. L. de Souza. 1982. Visual responses of South American fruit Anastrepha fraterculus and Mediterranean fruit flies, Ceratitis capitata, to colored rectangles and spheres. Environ. Entomol. 11: 1202-1210.

Drummond F., E. Groden \& R. Prokopy. 1984. Comparative efficacy and optimal positioning of traps for monitoring apple maggot flies (Diptera: Tephritidae). Enviromental Entomology 13: 232-235.

Féron M., P. Delanove \& F. Soria. 1958. L'elevage massif artificiel de Ceratitis capitata Wied. Entomophaga 3: 45-53.

Féron M. 1962. L'instinct de reproduction chez la mouche mediterranéenne des fruit Ceratitis capitata Wied (Dipt., Trypetidae). Comportement sexuel. Comportement de ponte. Revue de Pathologie Végétale et d'Entomologie Agricole de France 41: 1-129.

Freeman R. \& J. R. Carey. 1990. Interaction of Host stimuli in the Ovipositional Response of the Mediterranean fruit Fly (Diptera: Tephritidae). Environmental Entomology 19 (4): 1075-1080.
Katsoyannos B. I., G. Patsouras \& M. Vrekoussi. 1985. Effect of colour hue and brightness of artificial oviposition substrates on the selection of oviposition site by Dacus oleae. Entomologia Experimentalis et Applicata 38: 205-214.

Katsoyannos B. I. 1989. Response to shape, size and colour. In: Robinson A. S. \& G. Hooper, eds. World Crop Pests Fruit Flies, Vol. 3A. Elsevier, Amsterdam. Pp. 307-324.

Marchini L. \& R. J. Wood. 1983. Laboratory studies on oviposition and on the structure of the ovipositor in the Mediterranean fruit fly Ceratitis capitata (Wied). In: Cavalloro, R., ed. Fruit Flies of Economic Importance. A. A. Balkema, Athens. Pp. 113.

Nakagawa S., R. Prokopy, T. Wong, J. Ziegler, S. Mitchell, T. Urago \& J. Harris. 1978. Visual orientation of Ceratitis capitata flies to fruit models. Entomologia Experimentalis et Applicata 24: 193-198.

Owens E. \& R. Prokopy. 1984. Habitat background characteristics influencing Rhagoletis pomonella (Walsh) (Dipt., Tephritidae) fly response to foliar and fruit mimic traps. Zeitschrift fúr Angewandte Entomologie 98: 98-103.

Prokopy R. J. \& A. P. Economopoulos. 1975. Attraction of laboratory-cultured and wild Dacus oleae flies to sticky-coated Mc Phail traps of different colors and odours. Environmental Entomology 4:187-192.

Prokopy R. J. 1977 b. Attraction of Rhagoletis flies (Diptera: Tephritidae) to red spheres of different sizes. The Canadian Entomologist 109: 593-596.

Prokopy R. J., P. T. Mc Donald \& T. T. Y. Wong. 1984. Inter-population variation among Ceratitis capitata flies in host acceptance pattern. Entomol. Exp. Appli. 35: 65-69.

Quesada Allué L. A. 1994. La mosca del Mediterráneo. Guía de Laboratorio. Ed. por el Autor, Bs. As.

Quilici S. \& L. Rivry. 1996. Influence of some visual stimuli on the selection of oviposition site by Ceratitis (Pteandrus) rosa. In: Mc. Pheron, B. A. \& G.J. Steck, eds. Fruit Fly Pests. Lucie Press, USA. Pp. 59-65.

Sanders W. 1962. Das Verhalten der Mittelmeerfruchflige Ceratitis capitata Wied. bei der Eiablage. Zeitschrift für Tieerpsychologie 19: 1-28.

Sanders W. 1968 a. Die Eiablagehandlung der Mittelmeerfruchtlie Ceratitis capitata Wied. Ihre. Abhangigkert von grosse und Dichte der Fruchte. Zeitschrift für Tierpsychologie 25: 1-21.

Sanders W. 1968 b. Die Eiablagehandlung der Mittelmeerfruchtlie Ceratitis capitata Wied. Ihre. Abhangigkert von Farbe und Gliederung des Umfelder. Zeitschrift für Tierpsychologie 25: 588-607.

Souza H. M. L., O. H. D. Parvan \& I. D. Silva. 1984. Oviposition and alightment behaviour of Ceratitis capitata (Diptera: Tephritidae) on colored spheres. Rev. Bras. Entomol. 28 (1): $11-14$

Tanaka N. 1965. Artificial egging receptacles for three species of the Tephritidae Flies. Journal Economic Entomology 58 (1): $177-178$. 
